\title{
Anomalous transport of a classical wave-particle entity in a tilted potential
}

\author{
Rahil N. Valani ${ }^{1 *}$ \\ ${ }^{1}$ School of Mathematical Sciences, University of Adelaide, South Australia 5005, Australia
}

(Dated: October 28, 2021)

\begin{abstract}
A classical wave-particle entity in the form of a millimetric walking droplet can emerge on the free surface of a vertically vibrating liquid bath. Such wave-particle entities have been shown to exhibit hydrodynamic analogs of quantum systems. Using an idealized theoretical model of this wave-particle entity in a tilted potential, we explore its transport behavior. The integro-differential equation of motion governing the dynamics of the wave-particle entity transforms to a Lorenz-like system of ordinary differential equations (ODEs) that drives the particle's velocity. Several anomalous transport regimes such as absolute negative mobility (ANM), differential negative mobility (DNM) and lock-in regions corresponding to force-independent mobility, are observed. These observations motivate experiments in the hydrodynamic walking-droplet system for the experimental realizations of anomalous transport phenomena.
\end{abstract}

Introduction. When a biased force is applied to a particle having no net drift, one expects the particle to drift in the direction of the applied force. However, seemingly paradoxical behaviors have been observed in nonequilibrium systems that may result in anomalous response of the particle under applied bias. For example, absolute negative mobility (ANM) may arise where the particle responds with a net drift in a direction opposite to the applied bias. A less pronounced related phenomenon is differential negative mobility (DNM), where the particle drifts in the same direction as the applied bias but the drift speed of the particle decreases with increasing applied bias. ANM has been observed experimentally and theoretically in both quantum and classical systems 1]. Examples of quantum systems that exhibit ANM include quantum-well structures [2] and semiconductor superlattices [3, 4]. In classical systems, ANM has been mainly observed and investigated in systems driven by noise such as single [5]12] and interacting [1317. Brownian particles, while fewer works have studied ANM in deterministic systems. Examples of deterministic systems that exhibit ANM include vibrational motors [18], a particle with space-[19] and speed-dependent damping [20, a particle in a time-varying potential [21], a particle in a periodic double-well potential [22] and a particle in a traveling wave system [23]. Inspired by the emergence of ANM in deterministic systems, in this Letter we theoretically and numerically investigate the anomalous transport behavior of a self-propelled classical wave-particle entity in a tilted potential.

A liquid bath when vibrated vertically can support millimetric droplets on its free surface that walk horizontally while bouncing vertically $24 \sqrt{26}$. The walking droplet, also known as a walker, upon each bounce generates a damped localized standing wave on the fluid surface. It then interacts with these self-generated waves on subsequent bounces to propel itself horizontally. The droplet and its underlying wave field coexist as a wave-particle

\footnotetext{
* rahil.valani@adelaide.edu.au
}

entity; the droplet generates the underlying wave field which in turn guides the motion of the droplet. At large vibration amplitudes, the waves created by a walker decay very slowly in time and the walker's motion is not only influenced by the wave created on its most recent bounce, but also by the waves generated in the distant past, giving rise to memory in this hydrodynamic system. In the high-memory regime, walkers have been shown to mimic several hydrodynamic analogs of quantum systems. Some of these include orbital quantization in rotating frames [27, 29] and confining potentials [30 33], Zeeman splitting in rotating frames [34, 35, wave-like statistical behavior in both confined geometries 36 40 and open systems 41, tunneling across submerged barriers 42,44 and a macroscopic analog of spin systems [45]. Walkers have also been predicted to show anomalous twodroplet correlations [46, 47. Recently, efforts have also been made to develop a hydrodynamic quantum field theory for the walking-droplet system [48, 49]. A detailed review of hydrodynamic quantum analogs for walking droplets can be found in a recent review article by Bush and Oza [50].

In this Letter, we report anomalous transport behavior arising in a walking-droplet-inspired theoretical model that governs the dynamics of a one-dimensional waveparticle entity with a sinusoidal wave form in a tilted potential. We start by converting the integro-differential trajectory equation that governs the motion of the waveparticle entity into a system of Lorenz-like ordinary differential equations (ODEs) and perform a linear stability analysis to determine the stability of steady walking states. We then explore the different anomalous transport behaviors arising in both steady and unsteady walking regimes of the parameter space.

Theoretical model. As shown schematically in Fig. 1, consider a particle located at position $x_{d}$ moving horizontally with velocity $\dot{x}_{d}$ while continuously generating waves with prescribed spatial structure $W(x)$ that decay exponentially in time. The dimensionless equation of motion governing the horizontal dynamics of the particle is 


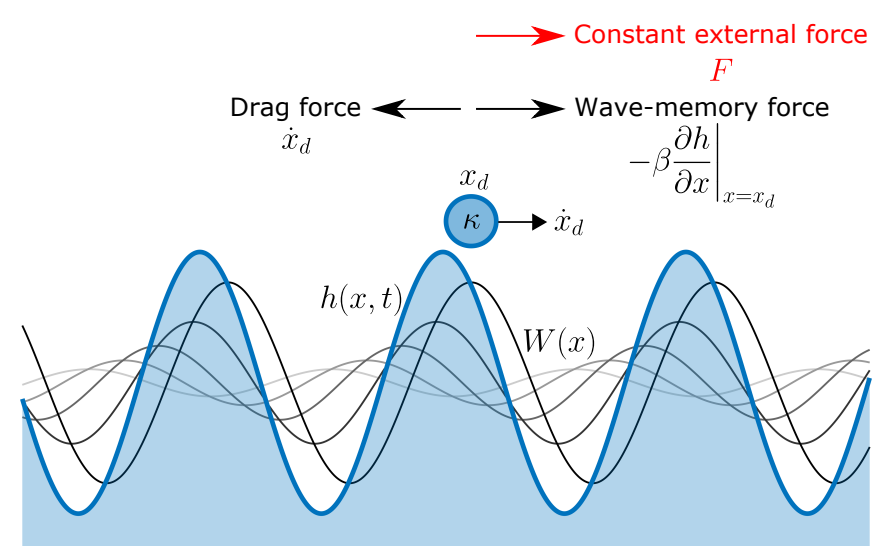

FIG. 1. Schematic of the one-dimensional self-propelled waveparticle entity. A particle of dimensionless mass $\kappa$ is located at $x_{d}$ and moving horizontally with velocity $\dot{x}_{d}$. The particle experiences a propulsion force, $-\beta \partial h /\left.\partial x\right|_{x=x_{d}}$, from its self-generated wave field $h(x, t)$ (blue filled area), an effective drag force $-\dot{x}_{d}$ and a constant external force $F$. The underlying wave field $h(x, t)$ is a superposition of the individual waves continuously generated by the particle along its trajectory. These individual waves (black and gray curves with the higher intensity of the color indicating the waves created more recently) are of spatial form $W(x)=\cos (x)$, and decay exponentially in time.

given by [51,

$$
\kappa \ddot{x}_{d}+\dot{x}_{d}=-\left.\beta \frac{\partial h}{\partial x}\right|_{x=x_{d}}+F .
$$

The left hand side of Eq. (1) comprises an inertial term $\kappa \ddot{x}_{d}$ and an effective drag term $\dot{x}_{d}$, where the overdot denotes differentiation with respect to time $t$. The first term on the right hand side captures the forcing on the droplet by the underlying wave field $h(x, t)$. This force is proportional to the gradient of the underlying wave field. The second term is an external constant force $F$ arising from a tilted potential $V(x)=-F x$. The shape of the wave field $h(x, t)$ is calculated through integration of the individual wave forms $W(x)$ that are continuously generated by the particle along its trajectory, giving

$$
h(x, t)=\int_{-\infty}^{t} W\left(x-x_{d}(s)\right) \mathrm{e}^{-(t-s)} \mathrm{d} s .
$$

Combining Eqs. (1) and 21, one obtains the integrodifferential equation

$$
\kappa \ddot{x}_{d}+\dot{x}_{d}=\beta \int_{-\infty}^{t} f\left(x_{d}(t)-x_{d}(s)\right) \mathrm{e}^{-(t-s)} \mathrm{d} s+F,
$$

where $f(x)=-W^{\prime}(x)$ is the negative gradient of the wave form and the prime denotes differentiation with respect to the argument $x$. The two parameters, $\kappa>0$ and $\beta>0$, follow directly from Oza et al. [51] and may be usefully interpreted as the ratio of inertia to drag and the ratio of wave forcing to drag respectively. This integrodifferential trajectory equation was derived by Oza et al.
[51] to describe the horizontal dynamics of a walking droplet by employing a Bessel function of the first kind and zeroth order, $W(x)=\mathrm{J}_{0}(x)$, wave form for the individual waves generated by the droplet on each bounce. The model was recently investigated by Valani et al. 52 . and Durey [53 by employing a simpler sinusoidal wave form $W(x)=\cos (x)$ and it was shown by Valani et al. [52] and Valani [54] that the integro-differential equation of motion can be transform to the following set of ODEs (see Supplemental Material [55] for a derivation):

$$
\begin{aligned}
& \dot{X}=\sigma(Y-X+F), \\
& \dot{Y}=-X Z+r X-Y, \\
& \dot{Z}=X Y-b Z .
\end{aligned}
$$

These ODEs are the Lorenz equations with an added constant term $F$ in the first equation [56, 57]. Here, $X=\dot{x}_{d}$ is the droplet's velocity, $Y=\beta \int_{-\infty}^{t} \sin \left(x_{d}(t)-\right.$ $\left.x_{d}(s)\right) \mathrm{e}^{-(t-s)} \mathrm{d} s$ is the wave-memory force and $Z=$ $\beta-\beta \int_{-\infty}^{t} \cos \left(x_{d}(t)-x_{d}(s)\right) \mathrm{e}^{-(t-s)} \mathrm{d} s$ is also related to the wave-memory forcing. Thus, the underdamped dynamics of an inertial particle of dimenionsionless mass $\kappa$ driven by a wave-memory force with coupling $\beta$, can alternatively be interpreted as the overdamped dynamics of a particle whose velocity $\dot{x}_{d}=X$ is driven by the Lorenz system with parameters $\sigma=1 / \kappa, r=\beta$ and $b=1$. For the simulation results presented in this Letter, the system of ODEs in Eq. (4) is solved in MATLAB using the inbuilt ODE solver ode45.

Steady solutions and linear stability analysis. To obtain steady walking solutions of the wave-particle entity, we start by finding fixed points of the Lorenz-like system presented in Eq. (4). This gives the equilibrium solutions $X_{0}=u, Y_{0}=u-F$ and $Z_{0}=u(u-F)$, where the constant velocity $u$ satisfies the cubic equation [55]

$$
u^{3}-F u^{2}-(\beta-1) u-F=0 .
$$

For $F>0(F<0)$ and $0<\beta<1$, there is one real positive (negative) solution to Eq. (5), while for $\beta>1$, one can conclude the existence of one real positive (negative) solution and either two or zero real negative (positive) solutions by invoking Descartes' rule of signs. To determine the stability of these steady walking solutions, one can perform a linear stability analysis by applying a small perturbation to the steady solutions [58. This results in the following characteristic equation for the growth rate $\lambda$ of small perturbations [55]:

$$
\begin{aligned}
& \kappa \lambda^{3}+(2 \kappa+1) \lambda^{2}+\left[\kappa\left(1+u^{2}\right)+2-\beta+u(u-F)\right] \lambda \\
& +1+3 u^{2}-\beta-2 u F=0 .
\end{aligned}
$$

The linear stability diagram in the $(\kappa, \beta)$ parameter space for a fixed $F=0.5$ is shown in Fig. 2(a). At small $\beta$ values, there is one stable steady walking solution corresponding to the wave-particle entity traveling in the direction of the applied force $F$. Above a critical value of 

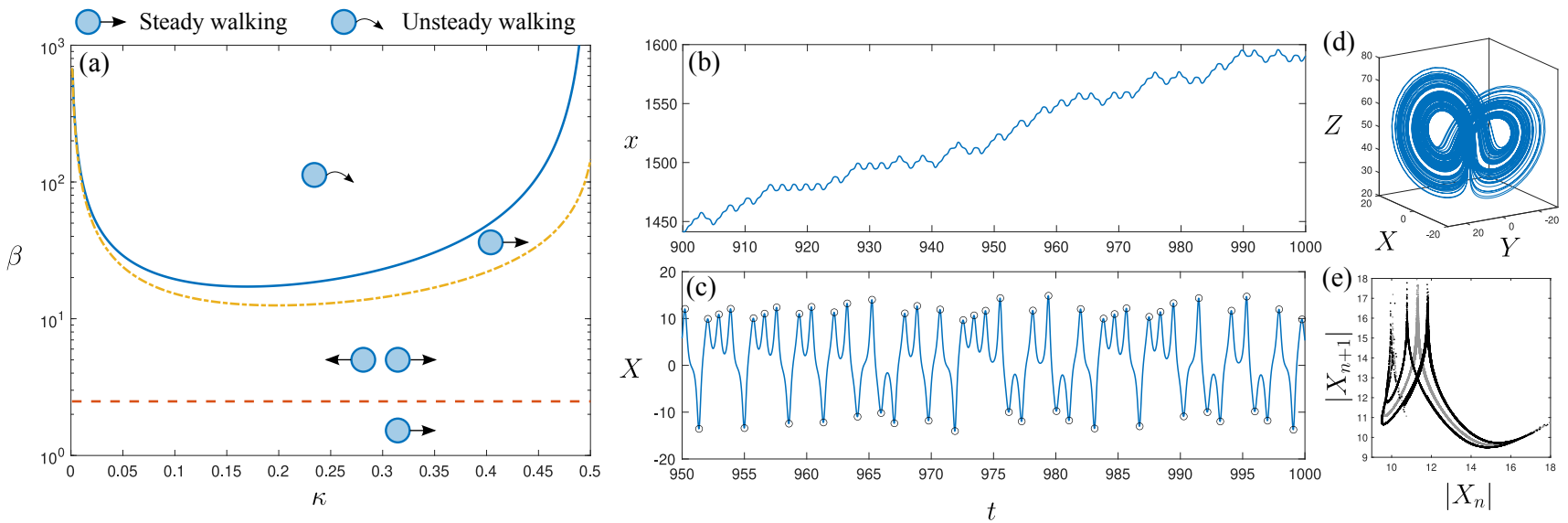

FIG. 2. (a) Linear stability diagram of the wave-particle entity in the $(\kappa, \beta)$ parameter space at $F=0.5$. Curves show parameter values when eigenvalues $\lambda$ of the dynamical system cross the $\operatorname{Re}(\lambda)=0$ line, indicating a change in stability. Chaotic dynamics in the unsteady regime of the $(\kappa, \beta)$ parameter space for a typical parameter value of $\kappa=0.3$ and $\beta=50$ is shown in (b)-(d). (b) shows the space-time trajectory, (c) shows the corresponding velocity time series and (d) shows the underlying strange attractor that drives the chaotic dynamics. The black curve in (e) shows the 1D return map of consecutive maxima (denoted by black circles in (c)) of absolute velocity $|X|$, while the gray curve shows the same for the classic Lorenz system with $F=0$.

$\beta$ (dashed red line) which is independent of $\kappa$, a stableunstable pair of steady walking solutions emerges corresponding to the wave-particle entity walking steadily in the direction opposite to the applied force $F$. This results in a total of three steady walking solutions: a higher speed stable and a lower speed unstable solution in the direction opposite to $F$, and a stable solution in the same direction as $F$. Thus, in this region of multistability, we observe ANM with the wave-particle entity walking steadily in a direction opposite to the applied bias. At higher $\beta$ (above the yellow dotted-dashed curve), the stable steady walking solution in the direction opposite to $F$ becomes unstable, followed by the instability of the steady walking solution in the direction of $F$ (above the solid blue line).

In the unsteady regime of the $(\kappa, \beta)$ parameter space at large $\beta$, we observe either chaotic dynamics or periodic oscillations in the particle's velocity $X$. Typical chaotic dynamics of the wave-particle entity driven by the underlying strange attractor in the unsteady regime is depicted in Fig. 2(b)-(d). As shown in Fig. 2(b), the particle seems to exhibits irregular diffusive-like behavior with a net drift in the direction of the force $F$. We note that in the absence of the force $F$, the particle has been demonstrated to exhibit diffusive-like behavior with no net drift 52. The time series of the particle's velocity $X$ in Fig.2(c), the underlying strange attractor in the $(X, Y, Z)$ phase-space in Fig. 2(d) and the 1D return map of the maxima in $|X|$ in Fig. 2(e), show similarities with the classic Lorenz system [56. We note that a doublecusp structure (black) is observed in the 1D return map here, as opposed to a single cusp structure (gray) observed for the classic Lorenz system. This single-cusp to double-cusp transition is due to the asymmetry introduced by the constant bias force $F$ which breaks the

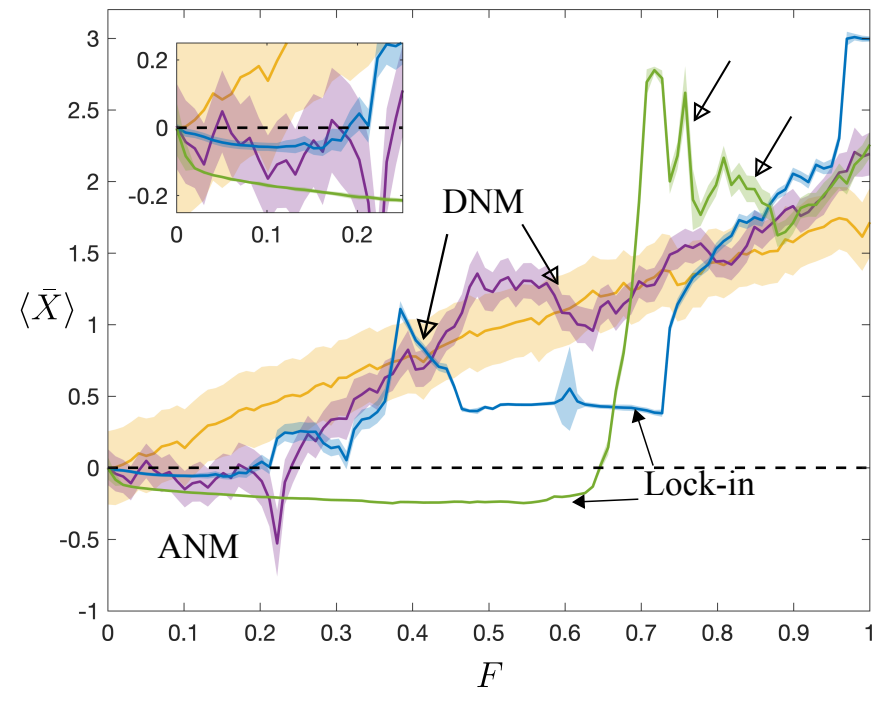

FIG. 3. Anomalous transport behavior. The average velocity $\langle\bar{X}\rangle$ of the particle against the applied constant force $F$ is shown for four sets of parameter values: $(\kappa, \beta)=(0.20,30)$ (yellow curve), $(0.17,67)$ (purple curve), $(0.25,100)$ (blue curve) and $(0.30,140)$ (green curve). The dashed horizontal line indicates $\langle\bar{X}\rangle=0$. The shaded region shows the standard deviation of $\bar{X}$ over the 1000 simulated trajectories.

degeneracy of the left and right walking states.

Anomalous transport in the unsteady regime. To investigate the particle's mobility in the unsteady regime, simulations were performed at fixed parameter values with $M=1000$ different random initial conditions. The initial values of the dynamical variables $X, Y$ and $Z$ were drawn randomly from a uniform probability distribution between -1 and 1 . A time-averaged particle velocity for a given initial condition was first cal- 

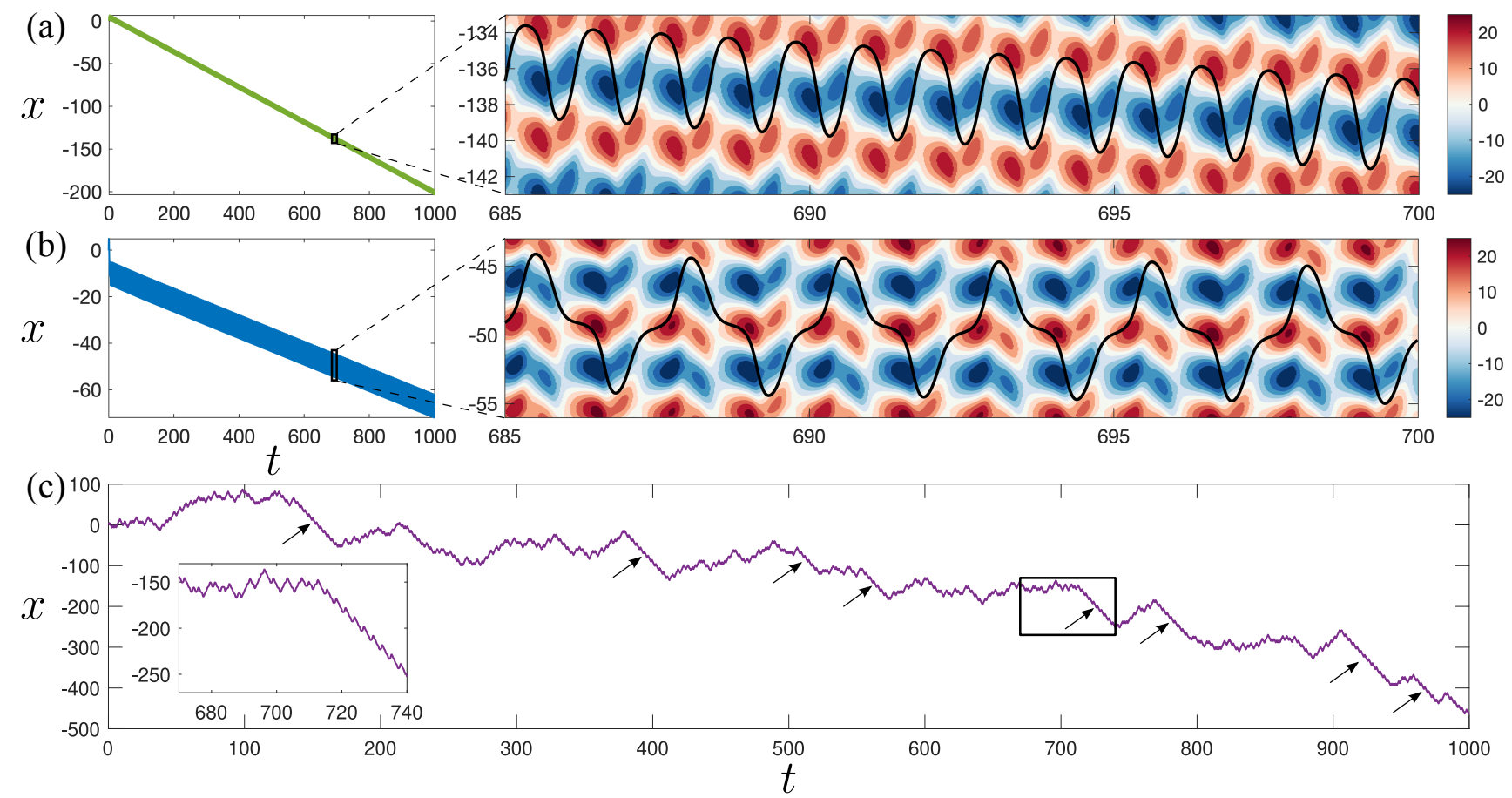

FIG. 4. Space-time trajectory of the particle during anomalous transport. Trajectory of the particle for ANM shown at (a) $(\kappa, \beta, F)=(0.30,140,0.2),(\mathrm{b})(0.25,100,0.1)$ and (c) $(0.17,0.67,0.22)$. The enlarged trajectory in panels (a) and (b) also show contours of the underlying wave field $h(x, t)$. The arrows in panel (c) show bursts of periodic motion in the opposite direction to the applied force.

culated as $\bar{X}_{j}=\sum_{i=1}^{N} X_{j}\left(t_{i}\right) / N$, which was then ensemble averaged over all the initial conditions to give $\langle\bar{X}\rangle=\sum_{j=1}^{M} \bar{X}_{j} / M$. This average velocity $\langle\bar{X}\rangle$ as a function of the applied force $F$ is shown in Fig. 3 for four different sets of $(\kappa, \beta)$ values showing the various transport behaviors observed in the unsteady regime. For the parameter set $(\kappa, \beta)=(0.20,30)$ (yellow curve), we find that $\langle\bar{X}\rangle$ increases almost linearly in the direction of the applied force as the magnitude of the applied force increases. This indicates normal mobility behavior that one finds in equilibrium systems. For the parameter sets $(\kappa, \beta)=(0.17,67)$ (purple curve), $(0.25,100)$ (blue curve) and $(\kappa, \beta)=(0.30,140)$ (green curve), we clearly see regions of ANM where $\langle\bar{X}\rangle<0$. We have observed two qualitatively different types of ANM in the unsteady regime: (i) the particle is undergoing periodic back-and-forth oscillations with a net drift in the direction opposite to $F$ (see Fig. 4(a)-(b)) and (ii) the particle's velocity is chaotic resulting in an irregular diffusivelike trajectory with a net drift in the direction opposite to $F$ (see Fig. 4(c)). In the periodic ANM regime at parameter values $(\kappa, \beta, F)=(0.30,140,0.2)$, the particle oscillates back-and-forth between two consecutive peaks of the underlying wave field along with a net drift in the direction opposite to $F$, while, at parameter values $(\kappa, \beta, F)=(0.25,100,0.1)$, the particle is able to cross one peak during back-and-forth oscillations and hence oscillates with twice the wavelength along with a slower net drift opposite to $F$. In the chaotic ANM at parame- ter values $(\kappa, \beta, F)=(0.17,67,0.22)$, although the spacetime trajectory exhibits irregular diffusive-like dynamics, we observe intermittent bursts of periodic drifts in the direction opposite to $F$ which results in an average motion opposite to the applied force. In addition to ANM, we also see in Fig. 3 regions of DNM which correspond to a negative slope in the curve when $\langle\bar{X}\rangle>0$, indicating that an increase in $F$ leads to decrease in $\langle\bar{X}\rangle$. We also encounter another anomalous transport behavior that we term lock-in regions. Here the average velocity $\langle\dot{X}\rangle$ stays almost constant for a range of $F$ values, indicating forceindependent mobility. In these lock-in regions, the particle is typically undergoing periodic back-and-forth oscillations with a net drift either in the direction of $F$ or opposite to $F$.

Conclusions. In this Letter, we have shown anomalous transport behaviors of a wave-memory driven particle under the presence of a constant external force. In the steady walking regime, ANM is observed in the multistable region where a stable steady walking solution is realized in the direction opposite to the applied force. In the unsteady walking regime, typically, the wave-particle entity undergoes either back-and-forth oscillations or irregular diffusive-like motion, with a net drift in the direction of the applied bias, but remarkably, regions of anomalous transport behaviors were observed where the wave-particle entity exhibits ANM, DNM and lock-in regions. The observations of anomalous transport reported in this Letter motivate experimental investiga- 
tions with walking and superwalking droplets [26, 59, 60] in a tilted potential where these behaviors may be realized in experiments. Moreover, the present study also motivates both theoretical and experimental investigations of other counterintuitive non-equilibrium phenom- ena, such as ratcheting effects and stochastic resonance, that may arise in the walking-droplet system.

Acknowledgements. I would like to thank David M. Paganin for useful discussions.
[1] R. Eichhorn, P. Reimann, B. Cleuren, and C. Van den Broeck, Moving backward noisily, Chaos 15, 026113 (2005)

[2] R. A. Höpfel, J. Shah, P. A. Wolff, and A. C. Gossard, Negative absolute mobility of minority electrons in GaAs quantum wells, Phys. Rev. Lett. 56, 2736 (1986).

[3] B. J. Keay, S. Zeuner, S. J. Allen, K. D. Maranowski, A. C. Gossard, U. Bhattacharya, and M. J. W. Rodwell, Dynamic localization, absolute negative conductance, and stimulated, multiphoton emission in sequential resonant tunneling semiconductor superlattices, Phys. Rev. Lett. 75, 4102 (1995)

[4] E. H. Cannon, F. V. Kusmartsev, K. N. Alekseev, and D. K. Campbell, Absolute negative conductivity and spontaneous current generation in semiconductor superlattices with hot electrons, Phys. Rev. Lett. 85, 1302 (2000)

[5] L. Machura, M. Kostur, P. Talkner, J. Łuczka, and P. Hänggi, Absolute negative mobility induced by thermal equilibrium fluctuations, Phys. Rev. Lett. 98, 040601 (2007)

[6] R. Eichhorn, P. Reimann, and P. Hänggi, Brownian motion exhibiting absolute negative mobility, Phys. Rev. Lett. 88, 190601 (2002)

[7] A. Słapik, J. Łuczka, P. Hänggi, and J. Spiechowicz, Tunable mass separation via negative mobility, Phys. Rev. Lett. 122, 070602 (2019).

[8] A. Słapik, J. Łuczka, and J. Spiechowicz, Negative mobility of a brownian particle: Strong damping regime, Commun. Nonlinear Sci. Numer. Simul. 55, 316 (2018).

[9] F. Deng, Y. Luo, Y. Fang, F. Yang, and C. Zeng, Temperature and friction-induced tunable current reversal, anomalous mobility and diffusions, Chaos Solit. Fractals 147, 110959 (2021)

[10] D. Speer, R. Eichhorn, and P. Reimann, Transient chaos induces anomalous transport properties of an underdamped brownian particle, Phys. Rev. E 76, 051110 (2007).

[11] J. Spiechowicz, P. Hänggi, and J. Łuczka, Coexistence of absolute negative mobility and anomalous diffusion, New J. Phys. 21, 083029 (2019)

[12] A. Slapik and J. Spiechowicz, Tunable particle separation via deterministic absolute negative mobility, Sci. Rep. 10, 16639 (2020)

[13] P. Reimann, R. Kawai, C. V. den Broeck, and P. Hänggi, Coupled brownian motors: Anomalous hysteresis and zero-bias negative conductance, Europhys. Lett. 45, 545 (1999)

[14] P. Reimann, C. Van den Broeck, and R. Kawai, Nonequilibrium noise in coupled phase oscillators, Phys. Rev. E 60, 6402 (1999).

[15] J. Buceta, J. M. Parrondo, C. Van den Broeck, and F. J. de la Rubia, Negative resistance and anomalous hysteresis in a collective molecular motor, Phys. Rev. E 61, 6287

\section{(2000)}

[16] B. Cleuren and C. V. den Broeck, Ising model for a brownian donkey, Europhys. Lett. 54, 1 (2001)

[17] M. Januszewski and J. Łuczka, Indirect control of transport and interaction-induced negative mobility in an overdamped system of two coupled particles, Phys. Rev. E 83, 051117 (2011)

[18] L. Du and D. Mei, Absolute negative mobility in a vibrational motor, Phys. Rev. E 85, 011148 (2012).

[19] C. Mulhern, Persistence of uphill anomalous transport in inhomogeneous media, Phys. Rev. E 88, 022906 (2013).

[20] W. Guo, L.-C. Du, Z.-Z. Liu, H. Yang, and D.-C. Mei, Uphill anomalous transport in a deterministic system with speed-dependent friction coefficient, Chin. Phys. B 26, 010502 (2017).

[21] B. S. Dandogbessi and A. Kenfack, Absolute negative mobility induced by potential phase modulation, Phys. Rev. E 92, 062903 (2015).

[22] Y. Luo, C. Zeng, and B.-Q. Ai, Strong-chaos-caused negative mobility in a periodic substrate potential, Phys. Rev. E 102, 042114 (2020)

[23] R. Chen, C. Wang, and Z. He, Abnormal transport behaviors in traveling wave system, Chaos Solit. Fractals 126, 116 (2019)

[24] Y. Couder, E. Fort, C.-H. Gautier, and A. Boudaoud, From bouncing to floating: noncoalescence of drops on a fluid bath, Phys. Rev. Lett. 94, 177801 (2005).

[25] Y. Couder, S. Protière, E. Fort, and A. Boudaoud, Dynamical phenomena: Walking and orbiting droplets, Nature 437, 208 (2005).

[26] R. N. Valani, A. C. Slim, and T. Simula, Superwalking droplets, Phys. Rev. Lett. 123, 024503 (2019).

[27] E. Fort, A. Eddi, A. Boudaoud, J. Moukhtar, and Y. Couder, Path-memory induced quantization of classical orbits, Proc. Natl. Acad. Sci. 107, 17515 (2010).

[28] D. M. Harris and J. W. M. Bush, Droplets walking in a rotating frame: from quantized orbits to multimodal statistics, J. Fluid Mech. 739, 444-464 (2014).

[29] A. U. Oza, D. M. Harris, R. R. Rosales, and J. W. M. Bush, Pilot-wave dynamics in a rotating frame: on the emergence of orbital quantization, J. Fluid Mech. 744, 404 (2014).

[30] S. Perrard, M. Labousse, E. Fort, and Y. Couder, Chaos driven by interfering memory, Phys. Rev. Lett. 113, 104101 (2014).

[31] S. Perrard, M. Labousse, M. Miskin, E. Fort, and Y. Couder, Self-organization into quantized eigenstates of a classical wave-driven particle, Nat. Commun. 5, 3219 (2014).

[32] M. Labousse, S. Perrard, Y. Couder, and E. Fort, Selfattraction into spinning eigenstates of a mobile wave source by its emission back-reaction, Phys. Rev. E 94, 042224 (2016).

[33] J. Montes, F. Revuelta, and F. Borondo, Bohr- 
Sommerfeld-like quantization in the theory of walking droplets, Phys. Rev. E 103, 053110 (2021)

[34] A. Eddi, J. Moukhtar, S. Perrard, E. Fort, and Y. Couder, Level splitting at macroscopic scale, Phys. Rev. Lett. 108, 264503 (2012)

[35] A. U. Oza, R. R. Rosales, and J. W. M. Bush, Hydrodynamic spin states, Chaos 28, 096106 (2018)

[36] D. M. Harris, J. Moukhtar, E. Fort, Y. Couder, and J. W. M. Bush, Wavelike statistics from pilot-wave dynamics in a circular corral, Phys. Rev. E 88, 011001 (2013).

[37] T. Gilet, Quantumlike statistics of deterministic waveparticle interactions in a circular cavity, Phys. Rev. E 93, 042202 (2016).

[38] P. J. Sáenz, T. Cristea-Platon, and J. W. M. Bush, Statistical projection effects in a hydrodynamic pilot-wave system, Nat. Phys. 14, 315 (2018).

[39] T. Cristea-Platon, P. J. Sáenz, and J. W. M. Bush, Walking droplets in a circular corral: Quantisation and chaos, Chaos 28, 096116 (2018).

[40] M. Durey, P. A. Milewski, and Z. Wang, Faraday pilotwave dynamics in a circular corral, J. Fluid Mech. 891, A3 (2020).

[41] P. J. Sáenz, T. Cristea-Platon, and J. W. M. Bush, A hydrodynamic analog of Friedel oscillations, Sci. Adv. 6 (2020).

[42] A. Eddi, E. Fort, F. Moisy, and Y. Couder, Unpredictable tunneling of a classical wave-particle association, Phys. Rev. Lett. 102, 240401 (2009).

[43] A. Nachbin, P. A. Milewski, and J. W. M. Bush, Tunneling with a hydrodynamic pilot-wave model, Phys. Rev. Fluids 2, 034801 (2017).

[44] L. Tadrist, T. Gilet, P. Schlagheck, and J. W. M. Bush, Predictability in a hydrodynamic pilot-wave system: Resolution of walker tunneling, Phys. Rev. E 102, 013104 (2020)

[45] P. J. Sáenz, G. Pucci, S. E. Turton, A. Goujon, R. R. Rosales, J. Dunkel, and J. W. M. Bush, Emergent order in hydrodynamic spin lattices, Nature 596, 58 (2021).

[46] R. N. Valani, A. C. Slim, and T. Simula, Hong-Ou-Mandel-like two-droplet correlations, Chaos
28, 096104 (2018).

[47] A. Nachbin, Walking droplets correlated at a distance, Chaos 28, 096110 (2018)

[48] Y. Dagan and J. W. M. Bush, Hydrodynamic quantum field theory: the free particle, Comptes Rendus. Mécanique 348, 555 (2020).

[49] M. Durey and J. W. M. Bush, Hydrodynamic quantum field theory: The onset of particle motion and the form of the pilot wave, Front. Phys. 8, 300 (2020).

[50] J. W. M. Bush and A. U. Oza, Hydrodynamic quantum analogs, Rep. Prog. Phys. (2020)

[51] A. U. Oza, R. R. Rosales, and J. W. M. Bush, A trajectory equation for walking droplets: hydrodynamic pilotwave theory, J. Fluid Mech. 737, 552 (2013).

[52] R. N. Valani, A. C. Slim, D. M. Paganin, T. P. Simula, and T. Vo, Unsteady dynamics of a classical particle-wave entity, Phys. Rev. E 104, 015106 (2021).

[53] M. Durey, Bifurcations and chaos in a Lorenz-like pilotwave system, Chaos 30, 103115 (2020).

[54] R. N. Valani, Lorenz-like systems emerging from an integro-differential trajectory equation of a onedimensional wave-particle entity, .

[55] See Supplemental Material at [URL will be inserted by publisher] for derivation of the Lorenz-like system of ODEs and additional details of the steady walking solution as well as the linear stability analysis.

[56] E. N. Lorenz, Deterministic Nonperiodic Flow, J Atmos Sci. 20, 130 (1963).

[57] A. A. Mishra and S. Sanghi, A study of the asymmetric malkus waterwheel: The biased lorenz equations, Chaos 16, 013114 (2006).

[58] S. Strogatz, Nonlinear dynamics and chaos : with applications to physics, biology, chemistry, and engineering (CRC Press, Boca Raton, 2019).

[59] R. N. Valani, J. Dring, T. P. Simula, and A. C. Slim, Emergence of superwalking droplets, J. Fluid Mech. 906, A3 (2021).

[60] R. N. Valani, A. C. Slim, and T. P. Simula, Stop-and-go locomotion of superwalking droplets, Phys. Rev. E 103, $043102(2021)$ 